\title{
Convívio e cuidado familiar na quarta idade: qualidade de vida de idosos e seus cuidadores
}

\author{
Living together and family care at the fourth age: quality of life for seniors and their caregivers
}

\section{Resumo}

É importante almejar qualidade de vida ou bem-viver aos idosos que já atingiram 80 ou mais anos de idade, com autonomia e independência. Com o avançar da idade, entretanto, torna-se mais frequente a dependência dos cuidados da família por parte dos idosos. Objetivo: Avaliar a dinâmica da família, a qualidade e o estilo de vida de idosos da quarta idade e de seus familiares cuidadores. Método: Estudo descritivo do qual participaram 100 idosos e seus cuidadores. Os dados foram coletados por meio do Questionário do Perfil da Família Cuidadora, o Family APGAR, Whoqol-Old, Whoqol-Breve e o Estilo de vida de Nahas. Resultado: A amostra de idosos caracterizouse por ser a maioria mulher, com idade média de 84 anos, portadora de hipertensão arterial e cardiopatias. Os cuidadores (67\%) viviam na casa dos próprios idosos, eram predominantemente mulheres, entre filhas e netas, sendo $20 \%$ idosos cônjuges de ambos os sexos. Embora os cuidadores tenham referido boa saúde e qualidade de vida, seu estilo de vida atingiu nível regular. Na ótica dos idosos, a família apresenta alta disfuncionalidade, embora tenham uma melhor qualidade de vida em alguns domínios e facetas. Conclusão: Com base na pesquisa, foi possível identificar a necessidade de redirecionar as políticas públicas e sociais a favor da rede de apoio à população idosa mais envelhecida, extensiva às famílias cuidadoras.

\section{Abstract}

It is important to get the quality of life or well being to the elderly who reached the fourth age - 80 years or more - with autonomy and independence. However, as people grow old, reliance on family care for the elderly becomes more frequent. Objective: This study aimed to assess family dynamics, quality of life and lifestyle of elderly at the fourth age and of their family caregivers. Method: Descriptive study involving 100 elderly and their caregivers. Data were collected by applying the Family Caregiver Profile Questionnaire, Family APGAR, Whoqol-Old, Whoqol-Bref and Nahas Lifestyle. Results: The elderly sample comprised mostly women, with mean age of 84
Lucia Takase Hisako Gonçalves ${ }^{1}$ Marinês Tambara Leite ${ }^{2}$ Leila Mariza Hildebrandt ${ }^{2}$ Silvana Cogo Bisogno² Sandra Biasuz ${ }^{2}$ Bruna Liége Falcade 2
Palavras-chave: Idoso de 80 anos ou mais. Assistência Domiciliar. Cuidados. Qualidade de Vida. Relações Familiares. Cuidadores.
Key words: Elderly aged 80 years and over. Home Nursing Care. Quality of Life. Family Relations. Caregivers.

\footnotetext{
Programa de Pós-graduação em Enfermagem. Universidade Federal do Pará. Belém, PA, Brasil.

2 Departamento de Ciências da Saúde. Universidade Federal de Santa Maria. Santa Maria, RS, Brasil.
} 
years, mostly of all with hypertension and heart diseases. Caregivers (67\%) lived in the elderly's household, were mostly women, daughters and granddaughters; $20 \%$ were elderly caregivers partners from both sexes. Although caregivers have reported good health and quality of life at a good level, their lifestyle was regular. According to the elderly, families have high dysfunctionality, although they had better levels of quality life in some areas and facets. Conclusion: Based on the research it was possible to identify the needs of redirecting to the public and social policies, in favor of a supporting network to the aged population and their family.

\section{INTRODUÇÃO}

Viver mais anos é uma aspiração das pessoas em qualquer sociedade. Para isso é importante que elas envelheçam com qualidade de vida, para atingir a quarta idade com autonomia e independência. Nesse sentido, avanços no campo da saúde e melhores condições de vida da população favorecem o aumento da expectativa de vida das pessoas. ${ }^{1}$ Quando se trata de idosos mais idosos, o estrato formado por indivíduos com 75 anos ou mais de idade apresentou maior crescimento relativo (49,3\%) nos últimos dez anos, quando comparado ao total da população idosa. ${ }^{2}$

O contingente populacional formado por indivíduos que chegaram à quarta idade - os octogenários - tem demonstrado atualmente que estão envelhecendo com mais autonomia e independência para o viver diário, mesmo que em equilíbrio lábil do estado de saúde. ${ }^{3}$ Isso pode, portanto, denotar melhor qualidade de vida dessa população.

O complexo conceito de qualidade de vida é definido pela Organização Mundial da Saúde (OMS) como a percepção que o indivíduo tem de sua posição na vida no contexto da cultura e do sistema de valores onde vive e em relação aos seus objetivos, expectativas, padrões e preocupações. É um conceito subjetivo que incorpora diversos aspectos da saúde física, do estado psicológico, das relações sociais, de crenças e sua relação com características destacadas no ambiente, além da dependência para os cuidados diários. ${ }^{4}$ Assim, qualidade de vida constitui um constructo que contempla extensa gama de aspectos da vida de uma pessoa que a faz autoavaliar como usufruindo ou não a sensação de bem-estar - ou seja, o quanto ela está ou não satisfeita com sua vida. Sendo subjetivo, o conceito depende de cada pessoa, de sua condição sociocultural, da idade e das aspirações pessoais, condições pelas quais o sujeito considera melhor ou pior, e oscila entre as dimensões física, psicológica e social. ${ }^{5}$

O processo de envelhecimento e sua vivência, portanto, constituem experiência particular a cada indivíduo. ${ }^{6}$ Afinal, várias são as circunstâncias vivenciadas de forma distinta pelas pessoas idosas, tais como as perdas ou não, cognitivas, físicas e mentais e as mudanças ou não na personalidade, na vida social e produtiva, que afetam de algum modo a autonomia e a independência para o viver diário e a qualidade de vida.

A dependência e falta de autonomia podem ser experienciadas de diferentes formas ao longo do ciclo vital. Para uma criança, são vistas como características próprias da fase da vida. No curso de uma doença, são percebidas como necessárias no período de transição para uma saúde melhor. Já na velhice, a falta de autonomia e a dependência são comumente eventos negativos e estressantes, que conduzem à baixa qualidade de vida, tanto para quem vivencia essa condição como para aqueles que estão no seu entorno.

A dependência de cuidados de outrem está relacionada à impossibilidade de os indivíduos se autocuidarem. Por motivos como a redução do custo da assistência hospitalar e institucional 
para idosos dependentes de cuidados, a atual tendência, em muitos países, incluindo o Brasil, é que os idosos permaneçam em suas casas sob os cuidados da família, independentemente do estado de saúde?

Sabe-se que não há modelo estável de família que possa contar sempre com a disponibilidade de seus membros para assistir às necessidades dos idosos dependentes. É mister atentar para o cuidado familiar, em que se avalia a estrutura e dinâmica da família em seu contexto sociocultural particular. Em geral, a qualidade de vida do idoso e de seu cuidador está entrelaçada e caminham juntas, uma vez que, habitualmente, o familiar cuidador desafia-se com dedicação ao cuidado da pessoa idosa. Além disso, ao cuidar do idoso, por longo tempo, expõe os cuidadores a risco de adoecimento, principalmente daqueles que são únicos e que assumem total responsabilidade pelo cuidado da pessoa idosa. Destaca-se, contudo, que outros integrantes da família podem e devem auxiliar em atividades complementares, daí serem chamados de cuidadores secundários. ${ }^{8}$

Tão importante quanto o bem-estar do idoso é o do seu cuidador, pois um interfere no equilíbrio da vida e da saúde do outro. Embora a família cuidadora não tenha seu papel reconhecido, tal lacuna deve ser considerada pelas políticas públicas e sociais voltadas para a população idosa. ${ }^{9}$

Considera-se importante que o familiar cuidador ten ha vida própria e não viva somente em função do idoso dependente. Isso só será possível se os cuidados ao idoso forem compartilhados entre mais pessoas e houver acompanhamento especializado e multidisciplinar por parte dos serviços sociais e de saúde. Isso pode permitir que as pessoas desenvolvam e exercitem hábitos de vida saudável, inserindo-se em programas de promoção da saúde e prevenção de enfermidades, centrados na realização regular de exercícios físicos, alimentação equilibrada e adoção de medidas profiláticas, como a imunização contra doenças imunopreveníveis. ${ }^{10}$
O objetivo desta pesquisa foi avaliar a dinâmica da família, a qualidade e o estilo de vida de idosos da quarta idade e de seus familiares cuidadores.

\section{MÉTODO}

Este estudo é parte de uma investigação multicêntrica denominada "Dinâmica da Família de Idosos mais Velhos (DIFAI): o convívio e cuidados na quarta idade", que foi desenvolvida nos contextos de Florianópolis-SC; Jequié-BA; Belém-PA; Palmeira das Missões-RS e Porto, Portugal. ${ }^{11}$

Neste artigo, foram trabalhados os dados de Palmeira das Missões-RS, os quais foram coletados entre setembro de 2009 e outubro de 2010. A investigação contou com uma amostra não probabilística, do tipo intencional, composta por 100 idosos, com 80 anos e mais de idade, dependentes de cuidados, residindo em ambiente doméstico-familiar e seus cuidadores, selecionados a partir da população vinculada às unidades básicas de saúde do município.

Como instrumentos de coleta dos dados, utilizaram-se o Questionário de Perfil da Família Cuidadora (QPFC), ${ }^{12}$ Family APGAR,${ }^{13}$ WhoqolOld $^{14}$ e Whoqol-Breve. ${ }^{15}$ O QPFC contém questões relativas a dados de identificação, variáveis sociodemográficas, estado de saúde e situações de cuidado de ambos, cuidador e idoso dependente. Este instrumento foi aplicado ao familiar cuidador.

Ao idoso aplicou-se o Family APGAR,,$^{13}$ com cinco questões simples que avaliam a dinâmica da família sobre adaptação intrafamiliar, convivência e comunicação, crescimento e desenvolvimento, afeto e dedicação da família, com opção de resposta em: quase sempre (dois pontos), algumas vezes (um ponto) e quase nunca (sem pontuação, ou seja, zero). Pelo valor da pontuação obtida, a família é classificada em três tipos: altamente funcional (7 a 10 pontos), moderadamente funcional (4 a 6 pontos) e 
acentuada disfuncionalidade ( $\left.\begin{array}{llll}0 & \text { a } & 3 & \text { pontos}\end{array}\right)$. Os estudos de validade e credibilidade do teste garantem a segurança na sua aplicação. ${ }^{13}$

No intuito de complementar os resultados da dinâmica familiar, avaliaram-se os aspectos correlatos, como qualidade e estilo de vida. Assim, aplicou-se ao idoso o instrumento de avaliação da qualidade de vida da Organização Mundial de Saúde (Whoqol), ${ }^{14-15}$ nas versões Breve e Old, já testadas, com adequados índices de validade e confiabilidade. ${ }^{15} \mathrm{O}$ Whoqol-Breve, com 26 questões, abrange quatro domínios: físico, psicológico, relações sociais e meio ambiente, com suas respectivas facetas. Cada questão oferece cinco opções de resposta (oscilando de 1 a 5 pontos), tipo escala de Likert. O Whoqol-Old compõe-se de 24 questões com facetas específicas: funcionamento do sensório, autonomia, atividades passadas, presentes e futuras, morte e morrer e intimidade. A pontuação dos resultados segue a lógica da anterior.

Para os familiares cuidadores utilizouse, além do Whoqol-Breve, a Escala de BemEstar de Nahas, ${ }^{16}$ que avalia o estilo de vida e os comportamentos que afetam a própria saúde e a qualidade das relações familiares. Inclui um conjunto de aspectos relativos à nutrição, atividades físicas, comportamentos preventivos para a saúde, relações sociais e controle de estresse que refletem atitudes, valores e oportunidades das pessoas. Cada um dos cinco aspectos contém três itens, perfazendo um total de 15 questões, com pontuação de zero a três. O máximo de pontos na escala é 45 , que corresponde a ótimo estilo de vida, e a pontuação mínima é zero, para inadequado estilo de vida.
Esse instrumento foi testado pelo próprio autor e apresenta validade e confiabilidade.

Para a análise dos dados, foi utilizado o programa estatístico computacional SPSS, versão 11.5. Os procedimentos estatísticos corresponderam à estatística descritiva (média, desvio-padrão e frequência absoluta).

O projeto foi submetido ao Comitê de Ética em Pesquisa com Seres Humanos da Universidade Federal de Santa Catarina (UFSC), aprovado e protocolado sob no $051 / 08$. Todos os participantes assinaram o Termo de Consentimento Livre e Esclarecido.

\section{RESULTADOS}

A amostra se constituiu de 100 idosos com idade de 80 e mais anos e de seus respectivos familiares cuidadores, localizados com base nos registros existentes nas unidades básicas de saúde da área urbana de Palmeira das Missões-RS (tabela 1). A média de idade dos idosos entrevistados foi de 84 anos, com predomínio de mulheres (63\%). A maioria dos octogenários (64\%) estudou até a $4^{a}$ série e 33\% deles não frequentaram o ensino formal. Em relação ao estado conjugal, predominam viúvos $(60 \%)$, seguidos de casados (35\%). A residência onde vivem é de propriedade dos idosos em $70 \%$ dos casos e dos cuidadores em $23 \%$.

Entre as doenças referidas pelos idosos, prevalecem a hipertensão arterial sistêmica em $60 \%$ e as cardiopatias, em 22\%. Em relação à funcionalidade familiar, os resultados apontam que $80 \%$ dos idosos possuem elevada disfuncionalidade. 
Tabela 1 - Caracterização dos idosos de 80 e mais anos, em âmbito domiciliar. Palmeira das MissõesRS, 2009-2010.

\begin{tabular}{|c|c|c|}
\hline Variável & $\mathrm{n}$ & $\%$ \\
\hline \multicolumn{3}{|l|}{ Idade por faixa etária } \\
\hline $80-85$ anos. & 68 & 68 \\
\hline $86-89$ anos & 23 & 23 \\
\hline 90 e mais anos & 9 & 9 \\
\hline Média de idade/desvio-padrão & \multicolumn{2}{|c|}{$84( \pm 3,94)$} \\
\hline \multicolumn{3}{|l|}{ Sexo } \\
\hline Masculino & 37 & 37 \\
\hline Feminino & 63 & 63 \\
\hline \multicolumn{3}{|l|}{ Escolaridade } \\
\hline Analfabeto & 33 & 33 \\
\hline Primário até $4^{\mathrm{a}}$ série & 64 & 64 \\
\hline Ginásio incompleto/completo & 2 & 2 \\
\hline Segundo grau incompleto/completo & 1 & 1 \\
\hline \multicolumn{3}{|l|}{ Estado Conjugal } \\
\hline Casado (e vive com cônjuge) & 35 & 35 \\
\hline Solteiro/separado & 5 & 5 \\
\hline Viúvo & 60 & 60 \\
\hline \multicolumn{3}{|l|}{ Professa religião } \\
\hline $\operatorname{Sim}$ & 86 & 86 \\
\hline \multicolumn{3}{|l|}{ A residência onde mora o idoso } \\
\hline Sua propriedade & 70 & 70 \\
\hline De seu cuidador & 23 & 23 \\
\hline Alugada/outros & 7 & 7 \\
\hline \multicolumn{3}{|l|}{ Doenças autorreferidas } \\
\hline Hipertensão arterial & 60 & 60 \\
\hline Cardiopatia & 22 & 22 \\
\hline Sequela de acidente vascular encefálico & 8 & 8 \\
\hline Diabetes mellitus & 8 & 8 \\
\hline Labirintite & 6 & 6 \\
\hline $\begin{array}{l}\text { Câncer (cólon, mama, pulmonar, bexiga, } \\
\text { estômago) }\end{array}$ & 4 & 4 \\
\hline Doença de Parkinson & 5 & 5 \\
\hline Doença de Alzheimer & 5 & 5 \\
\hline Outras & 11 & 11 \\
\hline \multicolumn{3}{|l|}{ Funcionalidade Familiar-Apgar (amplitude 0-10) } \\
\hline Boa funcionalidade (7-10) & 0 & 0 \\
\hline Moderada disfuncionalidade (4-6) & 20 & 20 \\
\hline Elevada disfuncionalidade $(0-3)$ & 80 & 80 \\
\hline \multicolumn{3}{|l|}{ Escore de qualidade de vida (amplitude 0-100) } \\
\hline WHOQOL-Breve - média e desvio-padrão & \multicolumn{2}{|c|}{$81,77 \pm 8,69$} \\
\hline WHOQOL-Old - média e desvio-padrão & \multicolumn{2}{|c|}{$78,48 \pm 2,84$} \\
\hline
\end{tabular}


Os resultados da avaliação da qualidade de vida dos idosos, obtidos por ambas as versões do Whoqol, apresentam-se semelhantes em bom nível, considerando que a pontuação desses instrumentos, em seus diferentes domínios, oscila de zero a 100 pontos. Quanto maior a média obtida, melhor é a qualidade de vida da população estudada. Assim, a qualidade de vida dos idosos, avaliada pelo Whoqol-Old em três faixas etárias diferentes, revela (tabela 2) que as pontuações das facetas "autonomia", "atividades passadas, presentes e futuras" e "participação social" tendem a diminuir com o avançar da idade. Já no domínio "intimidade", todas as faixas etárias apresentam baixa pontuação, enquanto no domínio "morte e morrer" a pontuação foi alta em todas elas.

Tabela 2 - Média e desvio-padrão dos escores das facetas do Whoqol-Old (amplitude 0-100), em idosos de 80 anos ou mais. Palmeira das Missões-RS, 2009-2010.

\begin{tabular}{lcccccc}
\hline \multirow{2}{*}{ Facetas Whoqol-old } & \multicolumn{7}{c}{ Faixa etária } \\
\cline { 2 - 7 } & \multicolumn{2}{c}{ Até 84} & \multicolumn{2}{c}{85 a 89} & \multicolumn{2}{c}{90 ou mais } \\
\cline { 2 - 7 } & Média & DP & Média & DP & Média & DP \\
\hline Funcionamento do sensório & 56,46 & $\pm 16,23$ & 51,41 & $\pm 20,20$ & 58,33 & $\pm 21,42$ \\
Autonomia & 65,10 & $\pm 36,27$ & 54,23 & $\pm 16,64$ & 50,00 & $\pm 25,58$ \\
Atividades passadas, & 63,65 & $\pm 11,59$ & 60,69 & $\pm 11,09$ & 61,11 & $\pm 14,91$ \\
presentes e futuras & 55,63 & $\pm 14,03$ & 49,40 & $\pm 16,17$ & 49,31 & $\pm 19,12$ \\
Participação social & 78,54 & $\pm 19,47$ & 72,78 & $\pm 22,22$ & 75,00 & $\pm 17,12$ \\
Morte e morrer & 33,54 & $\pm 36,00$ & 36,69 & $\pm 35,68$ & 16,67 & $\pm 33,66$ \\
Intimidade & & & & &
\end{tabular}

Os familiares cuidadores participantes da pesquisa (tabela 3) têm, em média, 50 anos $( \pm$ 14,53), e a maioria é do sexo feminino (89\%). Quanto à escolaridade, 41\% deles estudaram até o equivalente ao ensino fundamental. Sobre a ocupação, $22 \%$ dos cuidadores possuem outras atividades, além de cuidar do idoso. A maioria (67\%) mora no mesmo domić́lio do idoso e 33\% deles cuidam também de outros dependentes.
O grau de parentesco do familiar cuidador com o idoso prevalente é filha (50\%), seguido de cônjuge (19\%).

Quanto à autoapreciação da saúde, $84 \%$ dos cuidadores referem ter boa ou ótima saúde. Em relação à Escala de Nahas, os dados evidenciam um escore médio de 23,89 $( \pm 8,31)$, denotando que os cuidadores possuem estilo de vida regular. 
Tabela 3 - Caracterização do familiar cuidador principal de idosos de 80 e mais anos. Palmeira das Missões-RS, 2009-2010.

\begin{tabular}{|c|c|c|}
\hline Características & $\mathrm{n}$ & $\%$ \\
\hline Idade (Média e Desvio-padrão) & \multicolumn{2}{|c|}{$50,63 \pm 14,53$} \\
\hline \multicolumn{3}{|l|}{ Sexo } \\
\hline Masculino & 11 & 11 \\
\hline Feminino & 89 & 89 \\
\hline \multicolumn{3}{|l|}{ Estado conjugal } \\
\hline Casado (a) & 63 & 63 \\
\hline Solteiro & 15 & 15 \\
\hline Separado & 12 & 12 \\
\hline Viúvo (a) & 10 & 10 \\
\hline \multicolumn{3}{|l|}{ Escolaridade } \\
\hline Analfabeto & 6 & 6 \\
\hline Primário até $4^{\mathrm{a}}$ série & 41 & 41 \\
\hline Primeiro grau incompleto/completo & 23 & 23 \\
\hline Segundo grau incompleto/completo & 26 & 26 \\
\hline Superior incompleto/completo & 4 & 4 \\
\hline \multicolumn{3}{|l|}{ Ocupação } \\
\hline Trabalha & 22 & 22 \\
\hline Aposentado & 24 & 24 \\
\hline Cuidador de outros dependentes & 33 & 33 \\
\hline Mora com o idoso cuidado & 67 & 67 \\
\hline \multicolumn{3}{|l|}{ Autoapreciação da saúde } \\
\hline Ótima & 18 & 18 \\
\hline Boa & 66 & 66 \\
\hline Regular & 14 & 14 \\
\hline Ruim/péssima & 2 & 2 \\
\hline \multicolumn{3}{|l|}{ Parentesco do cuidador com o idoso } \\
\hline Cônjuge/irmã & 20 & 20 \\
\hline Filha & 50 & 50 \\
\hline Filho & 4 & 4 \\
\hline Neta & 9 & 9 \\
\hline Neto & 2 & 2 \\
\hline Sobrinha(o) & 2 & 2 \\
\hline Outros (cuidador contratado) & 13 & 13 \\
\hline \multicolumn{3}{|l|}{ Estilo de Vida (amplitude 0 - 45) } \\
\hline Média e desvio-padrão & & \\
\hline \multicolumn{3}{|l|}{ Escore qualidade de vida (amplitude 0-100) } \\
\hline WHOQOL-Breve - média e desvio-padrão & & \\
\hline
\end{tabular}


Quando comparada a qualidade de vida dos cuidadores com a dos idosos, a partir do Whoqol-Breve (tabela 4), a melhor média para ambos foi obtida no domínio "relações sociais"
- 84,15 e 75,42, respectivamente. A média mais baixa para os idosos foi no domínio "físico", com pontuação de 51,76. Já para os cuidadores, a média mais baixa foi no domínio "meio ambiente", com 71,24 pontos.

Tabela 4 - Média e desvio-padrão dos escores dos domínios do Whoqol-Breve, obtidos pelos cuidadores e idosos (amplitude de 0-100). Palmeira das Missões-RS, 2009-2010.

\begin{tabular}{lcccc}
\hline \multirow{2}{*}{ Domínio } & \multicolumn{2}{c}{ Média } & \multicolumn{2}{c}{ Desvio-padrão } \\
\cline { 2 - 5 } & Cuidador & Idoso & Cuidador & Idoso \\
\hline Físico & 82,61 & 51,76 & $\pm 14,05$ & $\pm 16,36$ \\
Psicológico & 74,84 & 63,23 & $\pm 10,02$ & $\pm 10,36$ \\
Relações sociais & 84,15 & 75,42 & $\pm 10,57$ & $\pm 9,20$ \\
Meio ambiente & 71,24 & 61,79 & $\pm 8,69$ & $\pm 10,37$ \\
\hline
\end{tabular}

\section{DISCUSSÃO}

Os dados apontam que há predomínio do sexo feminino na população estudada, o que está em consonância com a tendência da feminização da velhice, que cresce na razão direta com o avanço da idade. ${ }^{4,17,18}$ Outro dado evidenciado é o reduzido número de anos de ensino formal frequentado pelos idosos. Isto pode ter relação com a realidade pregressa de quem hoje conta com 80 ou mais anos de idade, ${ }^{18}$ em que o acesso a educação era restrito para esse contingente populacional.

Neste estudo identifica-se também a prevalência de afecções crônico-degenerativas, como a hipertensão e as cardiopatias, o que é um panorama epidemiológico comum às populações longevas. ${ }^{3,4,19}$ Destaca-se que o aumento da longevidade expõe a pessoa idosa a viver com enfermidades crônicas por um longo período, o que pode comprometer sua qualidade de vida. Essa condição exige políticas públicas de atenção que possibilitem a oferta de cuidados continuados e integrados às pessoas idosas, ${ }^{8,10}$ no intuito de preservar a capacidade funcional e a autonomia desses sujeitos. Em relação aos cuidadores, observa-se que os idosos estão sendo majoritariamente cuidados por mulheres. Este dado é semelhante a estudos divulgados na literatura que debate essa temática. ${ }^{20-22}$

Identifica-se que a maior parte das residências é de propriedade dos idosos. Assim sendo, a coabitação do cuidador com o idoso parece ser uma estratégia utilizada para beneficiar tanto os filhos quanto os idosos, em função das demandas de cuidado por parte destes e pelas necessidades financeiras dos familiares cuidadores. ${ }^{18}$ Ressaltase que, no caso desta investigação, corresidência era um dos critérios de inclusão dos sujeitos no estudo.

No intuito de reforçar, um estudo ${ }^{23}$ apontou que a corresidência é alternativa adotada pelas famílias para favorecer tanto as gerações mais jovens empobrecidas como as mais velhas, que necessitam de convívio e cuidados, podendo, no caso do Brasil, expressar melhoria nas condições de vida de ambos. Ainda se enfatiza a presença do cônjuge $(20 \%)$ entre os cuidadores, denotando que idosos estão cuidando de idosos. Esses dados são similares a outros estudos, os quais identificaram que houve aumento de cuidadores 
idosos, cônjuges de ambos os sexos, exercendo o encargo do cuidado de seus parceiros. ${ }^{24-30}$

A avaliação da qualidade de vida dos idosos, com a utilização do Whoqol Breve e Old, mostra bom nível de pontuação. Ao distribuir os escores obtidos nos estratos etários (8084, 85-89, 90 e +), identificam-se diferenças sensíveis entre as seis facetas, cujas variações não guardam necessariamente regularidade com o avançar da idade, ${ }^{27}$ denotando a manifestação da individualidade do idoso mesmo em idades mais avançadas. Com isso, é possível avaliar as especificidades das percepções mais positivas ou negativas dos idosos nas facetas de qualidade de vida.

Nesta investigação, destaca-se especialmente a faceta "intimidade", que apresenta baixo escore em todas as faixas etárias dos idosos participantes, o que pode ser atribuído à condição destes serem em boa parte viúvos, solteiros ou separados. Sobre a faceta "participação social" verifica-se que, embora a pontuação seja semelhante para os diferentes estratos etários, os idosos tendem a restringir seus contatos familiares e sociais, na medida em que a idade avança. Por sua vez, a faceta "morte e morrer" apresenta uma pontuação que pode ser considerada alta nos três estratos, indiferentemente de ser menos ou mais velho, inferindo-se que os idosos percebem essa faceta da qualidade de vida de modo natural. Observa-se, porém, tendência a diminuir os escores da qualidade de vida com o avançar da idade, sobretudo nas facetas que têm relação com a funcionalidade, já observadas em outros estudos, ${ }^{20,28}$ o que alerta para se considerar os efeitos da idade.

Os dados relativos aos cuidadores participantes deste estudo mostram certo comprometimento do convívio social, como se observa na avaliação da qualidade de vida, especialmente nos domínios "psicológico" e "meio ambiente", que apresentam escores menores. Por isso, na prática, é necessário articular redes locais de suporte social a partir dos serviços disponíveis na comunidade, para manter a inclusão social das famílias cuidadoras de idosos. ${ }^{27}$
Quanto aos aspectos relacionados à saúde dos cuidadores, embora a maioria tenha referido que seja boa ou ótima, os resultados da avaliação do estilo de vida mostram um nível regular. Isso possivelmente se deve ao estresse e a sobrecarga da tarefa de cuidar, restando-lhes pouco tempo para o cuidado de si, acrescido do desgaste pelo próprio envelhecimento, o que foi demonstrado também por outras pesquisas. ${ }^{26,27,31,32}$

Sobre a dinâmica de funcionalidade familiar, obtida com a aplicação do APGAR junto aos idosos, os resultados apontam alta disfuncionalidade. Isto parece ter relação com as exigências de cuidado requeridas pelo idoso com afecções crônicas e outros agravos, resultando em alguma forma de estresse na família, como a falta de adaptabilidade às mudanças de papéis de seus membros, aos novos estilos de relações intrafamiliares e às próprias relações de cuidado. Tal resultado remete à necessidade de intervenção junto ao familiar pela equipe de saúde, sobretudo de enfermeiros, que está envolvida na administração das dificuldades familiares em lidar com o cuidado ao idoso.

Nesse contexto, os recursos familiares podem ser pouco adequados para responder às necessidades de cuidado do idoso e/ou sinalizar a existência de áreas vulneráveis no contexto familiar. Isso pode interferir na habilidade da família em encontrar estratégias e recursos para desenvolver o papel de cuidadora. ${ }^{13,15,22}$ Por outro lado, as percepções pessoais dos membros de uma família acerca de certas variáveis, como qualidade de vida e estilo de vida, podem influir na dinâmica familiar, fazendo-a mais ou menos adaptativa, mais ou menos funcional, em face da situação que se apresenta como fato novo a enfrentar: um membro idoso que adoece e se torna cada dia mais frágil e dependente, exigindo cuidados e criando impactos sobre as relações intrafamiliares.

Guardadas as limitações do estudo, cujos dados foram extraídos de amostra por conveniência, o que impede sua generalização, seus resultados representam subsídios úteis à prática da saúde da família. 


\section{CONCLUSÃO}

O convívio doméstico e cuidados na quarta idade afetam a funcionalidade da dinâmica familiar, o que se reflete em percepções de nível regular ou mediano de qualidade de vida e estilo de vida dos cuidadores, além da dos próprios idosos nos diferentes domínios e facetas. Tal constatação implica redirecionamentos imprescindíveis nas políticas públicas e sociais a

\section{REFERÊNCIAS}

1. Instituto Brasileiro de Geografia e Estatística. Indicadores Sociodemográficos e de Saúde no Brasil 2009. Estudos \& Pesquisas: informação demográfica e socioeconômica n. 25. Rio de Janeiro: IBGE; 2009.

2. Instituto Brasileiro de Geografia e Estatística. Perfil dos idosos responsáveis pelos domicílios no Brasil 2000. Estudos \& Pesquisas: informação demográfica e socioeconômica n. 9. Rio de Janeiro: IBGE; 2002.

3. Veras RP, Caldas CP. A promoção da saúde de uma população que envelhece. In: Dallepiane LB, organizador. Envelhecimento humano: campo de saberes e práticas em saúde coletiva. Ijuí, RS: Unijuí; 2009. p. $57-80$.

4. Organização Mundial da Saúde. Envelhecimento ativo: uma política de saúde. Brasília: OPAS; 2005.

5. Dalla Vecchia R, Ruiz T, Bocchi SCM, Corrente JE. Qualidade de vida na terceira idade: um conceito subjetivo. Rev Bras Epidemiol 2005;8(3):246-52.

6. Chachamovich E, Trentini C, Fleck MPA. Qualidade de vida em idosos: conceituação e investigação. In: Neri AL, organizador. Qualidade de vida na velhice: enfoque multidisciplinar. Campinas: Alínea; 2007. p. 61-81.

7. Karsch UM. Idosos dependentes: famílias e cuidadores. Cad. Saúde Pública 2003:19(3):861-6.

8. Gonçalves LHT, Alvarez AM, Sena ELS, Santana LWS, Vicente FR. Perfil da família cuidadora de idoso doente/ fragilizado do contexto sociocultural de Florianópolis, SC. Texto Contexto Enferm 2006;15(4):570-7.

9. Garbin CAS, Sumida DH, Moimaz SAS, Prado RL, Silva MM. O envelhecimento na perspectiva do cuidador de idosos. Ciênc Saúde Coletiva 2010;15(6):2941-8. favor da rede de apoio à população idosa mais envelhecida e suas famílias cuidadoras.

A família cuidadora deve ser compreendida muito mais do que uma parceira; deve ser uma unidade de cuidados como cliente ou usuária dos serviços sociais e de saúde. Conhecer e acompanhar tal demanda emergente é fundamental para construir uma sociedade mais digna e acolhedora para a população longeva.

10. Leite MT. Envelhecimento humano: novas e velhas demandas no campo da saúde. Dallepiane LB, organizador. Envelhecimento humano: campo de saberes e práticas em saúde coletiva. Ijuí, RS: Unijuí; 2009. p. 95-106.

11. Gonçalves LHT, Santos SMA, Alvarez AM, Girondi JBR, Schier J, et al. A dinâmica da família de idosos mais idosos: o convívio e cuidados na quarta idade (DIFAI) [Projeto Edital 14/2008; Processo 474154/2008-4]. Brasília: MCT/CNPq; 2008.

12. Fratoni MM, Nassar SM, Goncalves LHT. Questionário de perfil da família cuidadora (QPFC). Software computacional. Florianópolis: GESPI/ UFSC; 2004

13. Duarte YAO. Familia: rede de suporte ou fator estressor. A ótica de idosos e cuidadores familiares [tese de Doutorado]. São Paulo: Escola de Enfermagem da Universidade de São Paulo; 2001.

14. World Health Organization; WHOQOL Group. WHOQOL: Measuring Quality of Life. Programme on mental health. Geneva: WHO; 1993.

15. Fleck MPA, coordenador. Versão em português do instrumento de avaliação de Qualidade de Vida (WHOQOL). 1998 [acesso em 12 nov 2011]. Disponível em: www.ufrgs.br/psiq/whoqol84.html

16. Nahas MV, Barros MVG, Francalacci V. O pentáculo do bem-estar: base conceitual para avaliação do estilo de vida de indivíduos ou grupos. Rev Bras Ativ Fís Saúde 2000;5(2):48-59.

17. Camarano AA, organizador. Os novos idosos brasileiros muito além dos 60? Rio de Janeiro: IPEA; 2004.

18. Lima LCV, Bueno CMLB. Envelhecimento e gênero: a vulnerabilidade de idosas no Brasil. Rev Saúde Pesquisa 2009;2(2):273-80. 
19. Falcade BL, Leite MT, Hildebrandt LM, Kirchner RM, Silva LAA, Gonçalves LHT. Octogenários em residências unipessoais: enfoque sobre a qualidade de vida e condições de saúde. R Enferm UFSM, 2011;1(3):386-93.

20. Joia LC, Ruiz T, Donalisio MR. Condições associadas ao grau de satisfação com a vida entre a população de idosos. Rev Saúde Pública 2007;41(1):131-8.

21. Inouye K, Pedrazzani ES, Pavarini SCI. Octogenários e cuidadores: perfil sócio-demográfico e correlação da variável qualidade de vida. Texto Contexto Enferm 2008;17(2):350-7.

22. Ferreira CG, Alexandre TS, Lemos ND. Fatores associados à qualidade de vida de cuidadores de idosos em assistência domiciliária. Saúde Soc 2011;20(2):398-409.

23. Camargos MCS. Estimativas de expectativa de vida livre de e com incapacidade funcional: uma aplicação do método de Sullivan para idosos paulistanos, 2000 [dissertação de Mestrado]. Belo Horizonte: Centro de Desenvolvimento e Planejamento Regional da Universidade Federal de Minas Gerais; 2004.

24. Pedrazzi EC. Arranjo domiciliar e apoio dos familiares aos idosos mais velhos [dissertação de Mestrado]. Ribeirão Preto: Escola de Enfermagem da Universidade de São Paulo; 2008.

25. Pimenta GMF, Costa MASMC, Gonçalves LHT, Alvarez AM. Perfil do familiar cuidador de idoso fragilizado em convívio doméstico da Grande
Região do Porto, Portugal. Rev Esc Enferm USP 2009;43(3):609-14.

26. Gonçalves LHT, Costa MAM, Martins MM, Nassar SM, Zunino R. The family dynamics of the elder elderly in the context of the Porto, Portugal. Rev Latino-Am Enferm 2011;19(3):458-66.

27. Rodrigues SLA, Watanabe HAW, Derntl AM. A saúde de idosos que cuidam de idosos. Rev Esc Enferm USP 2006;40(4):493-500.

28. Farenzena WP, Argimon IL, Moriguchi E, Portuguez MW. Qualidade de vida em um grupo de idosos de Veranópolis. Rev Kairós 2007;10(2):225-43.

29. Ducharme F, Lévesque L, Lachance L, Zarit S, Vézina J, Gangbè M, et al. Older husbands as caregivers of their wives: a descriptive study of the context and relational aspects of care. Int J Nurs Stud 2006;43(5):567-79.

30. Melo G. A experiência vivida de homens (cônjuges) que cuidam de mulheres com demência. Rev Bras Geriatr Gerontol 2009;12(3):319-30.

31. Oliveira DC, Carvalho GSF, Stella F, Higa CMH, D’Elboux MJ. Qualidade de vida e sobrecarga de trabalho em cuidadores de idosos em seguimento ambulatorial. Texto Contexto Enferm 2011;20(2):23440.

32. Moreira PHB, Mafra SCT, Pereira ET, Silva VE. Qualidade de vida de cuidadores de idosos vinculados ao Programa Saúde da Família - Teixeiras, MG. Rev Bras Geriatr Gerontol 2011;14(3):433-40. 